Voix et Images

voixetimages

\title{
Les belles solitudes
}

\section{André Brochu}

Volume 15, numéro 1 (43), automne 1989

Jacques Poulin

URI : https://id.erudit.org/iderudit/200827ar

DOI : https://doi.org/10.7202/200827ar

Aller au sommaire du numéro

\section{Éditeur(s)}

Université du Québec à Montréal

\section{ISSN}

0318-9201 (imprimé)

1705-933X (numérique)

Découvrir la revue

\section{Citer cet article}

Brochu, A. (1989). Les belles solitudes. Voix et Images, 15(1), 137-146.

https://doi.org/10.7202/200827ar d'utilisation que vous pouvez consulter en ligne.

https://apropos.erudit.org/fr/usagers/politique-dutilisation/ 


\section{Poésie}

\section{Les belles solitudes}

\section{par André Brochu, Université de Montréal}

N'était la pénurie des lecteurs, tout irait bien dans le petit monde des lettres, et en particulier de la poésie. Les idéologies, les écoles, les chapelles elles-mêmes se sont sabordées. Chacun peut désormais cultiver sa différence en toute sérénité. On voit s'áffirmer une collection vaste et diverse de moi forts - comme autant de belles solitudes. Le je est omniprésent, même quand le poème se donne des prétentions cosmiques ou mystiques. La fonction expressive retrouve les droits dont la modernité l'avait spoliée. Parions que cette situation ne durera guère. La chasse au lecteur s'accommode mal de la joyeuse et démocratique anarchie présente.

Chemins vacants, de Guy Ducharme ${ }^{1}$, est un premier recueil. Les poèmes, plutôt brefs et assez peu nombreux, répartis en trois sections, communiquent tout de suite l'impression d'un langage très sûr, direct comme une confidence et pourtant inépuisable; un langage gonflé de mystère tout humain, sans rien d'aliénant. Aucune esbroufe. C'est un homme qui dit sa vie partagée entre les tentations contraires du clos et de l'ouvert, du repli et de l'éparpillement, de la chambre et de la route, et ces contraires passent l'un dans l'autre, se résolvent en images synonymes:

fixe

vaste comme un ail

cette lune-là 
est le centre où tout se fond

lèvres chaudes et dents scellées

est le point

À la fois œil et bouche fermée, point final et lieu d'ouverture, la lune est tout humaine et pourtant, faste minéral. Elle est la chair tendre et l'étau des dents.

Le langage, lui aussi, est fait de tensions entre les contraires. Il est tout à la fois limpide et obscur, quotidien et évocateur d'une profonde sagesse vécue. Les images ne visent pas à l'épate; toujours vraies, elles ne libèrent leur charge d'émotion que si on les accueille en soi et on leur accorde la réflexion qu'elles requièrent. Par exemple:

Le jour comme un ouf

rabattu sur les toits

le ciel

si présent qu'il habille

Le monde est couvé, couvert, ramené à la dimension d'un simple désir humain, mais ce désir a l'ampleur du paysage, s'étend jusqu'au ciel dont il détermine la préserce et l'amitié.

Chemins vacants, c'est le monde possédé dans quelques mots détournés sans tapage de leur sens, pour leur faire prendre le cours de toute chose.

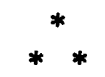

Gérald Godin, dans Poèmes de route ${ }^{2}$, continue de pratiquer cette poésie qu'on pourrait bien qualifier de carnavalesque puisqu'elle associe, à cette charge d'émotion et de générosité qui lui est habituelle, un côté gavroche, goguenard qui la tient loin des lyrismes convenus. Le titre, d'emblée, nous oriente vers la souriante sagesse populaire en assimilant les poèmes à des pommes de route qui jalonnent fort modestement la destinée humaine.

Souvent inspirée des menues circonstances de la vie, la poésie de Godin est toujours surprenante de simplicité et de vérité, grâce au parti qu'elle sait tirer de détails évocateurs:

mon propriétaire prononçait ses $r$ comme des gue

exemple:

monsieur Géguald Godin

ce qui faisait bien guigue

la grände Michèle Monroe

dans le motel de tous mes échecs à Hull

je me guéfugiais dans les toilettes

pour essayer de comprendre

ce qui se passait

sans guésultat aucun 
Ces vers terminent un assez long poème, d'une seule coulée, qui évoque les divers faits et gestes d'une existence décevante. Y revient comme un leitmotiv l'énoncé sans aucun résultat, modulé parfois en sans résultat connu. La bouffonne variante finale donne une poignante expansion de sens au constat désenchanté.

Qu'il parle de lui-même, de l'amour, de la vie qu'il lui reste à vivre, des mots expressifs du petit peuple, de la Terre qui se meurt, Gérald Godin reste une conscience vive de notre temps, qu'il saisit à travers ses manifestations les plus immédiates et les plus irrécusables.

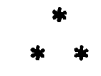

Renaud Longchamps a obtenu le prix Émile-Nelligan pour un livre intitulé Légendes suivi de Sommation sur l'histoire ${ }^{3}$. Comme tous les recueils de Longchamps, celui-ci comporte d'indéniables qualités. Le sérieux, d'abord et surtout. Malgré la fréquence du je, on n'est pas en présence de l'expression d'états d'âme fugitifs mais d'une poésie où la réflexion, quelle que soit la difficulté d'en cerner la problématique, joue un rôle important. Ce qui se joue $i c i$, précise le texte de présentation en page couverture, c'est la survie de l'espèce et le poète rassemble toutes ses énergies pour dire l'énervement de la vie, la nécessité de penser juste parmi le concert des voix neutres. L'ampleur du propos est confirmée par les titres de section qui évoquent Würm (la quatrième glaciation, entre 100000 et 15000 ans avant notre ère) et ces vastes thèmes que sont le Territoire ou l'Histoire.

Mais le registre épique qu'appelle le traitement de tels sujets est aussi absent que le registre lyrique qui accompagne normalement le je (et qui serait, certes, déplacé ici). À vrai dire, malgré de belles réussites çà et là - images suggestives, qui donnent à voir et à penser - il m'est difficile d'accorder une attention soutenue à ces poèmes, qui me semblent manquer singulièrement d'unité; qui se développent au gré d'une inspiration passablement arbitraire. D'une strophe à l'autre, on est transporté dans des espaces de sens peu compatibles avec ceux qu'on a quittés; aucune isotopie ne semble s'instaurer, qui permettrait au lecteur de laisser s'accomplir en lui le poème. Le fait peut même être observé, souvent, à l'intérieur d'une même strophe:

\section{Rien \\ rien de plus que l'amas \\ le repos complet dans un mouchoir}

où la touche finale, tout à fait triviale, est réfractaire à la suggestion, décourage tout sentiment poétique. Le lecteur ne peut, au long du recueil, que se familiariser avec les mots récurrents - os, héron, loi, corps, matière, désir, poussière, etc. - mais sans espoir de prendre pied dans cet univers qui, plus encore que le lisible, semble bien refuser le sens - hélas! à son grand détriment. 


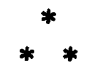

Dans Apprendre à écrire, Hugues Corriveau 4 décrit l'amour comme un commencement absolu. Fréquemment, le poème peint l'aube ou le soleil levant, vu de derrière la vitre; le poète s'adonne aux premiers gestes du jour, boit son café, tient la tasse chaude comme un crâne entre ses mains, éprouve la peur de vivre. Et la femme fait corps avec l'angoisse, avec le désir. Elle est comme la mère, être des origines; omniprésente, jamais désignée par son nom. D'ailleurs la mère était femme elle aussi, femme enfantée, elle était l'amour. Face au jour, donc, il faut apprendre à vivre, à partir d'un sentiment purement matériel d'exister, d'un affect brut où rien n'est encore bon ni mauvais, ni beau ni laid. Toute chose a sa pertinence propre: manger, mais aussi vomir. Le poète veut manger le monde, car rien ne distingue encore ce dernier de la présence nourricière. Le rapport à l'autre est celui du nourrisson à la mère:

les embryons les fretus les nouveau-nés

se mettent en place dans la torpeur de ma tête

certains bruits malveillants certaines peurs nettes

lisses comme des ongles, froides comme des os

je mangerai l' univers avant d'aller dehors

je resterai encore un peu ici dans la dolence

en effet le matin lourd va m'étonner

Tout est chair, tout est corps. Le matérialisme de Corriveau n'est pas une option idéologique mais une conviction spontanée. Tout se passe dans la sphère du moi-ici-maintenant où il y a des retournements d'estomac, le craquement des os, où la femme est odeur de vulve, où les mots captent la totale capacité des sens et donnent aux choses du monde / l'exactitude de leur forme. Dans ce monde habité, nullement métaphysique, le désir combat la peur de vivre et l'amour vainc raisonnablement la solitude. Une image le dit, en quelques mots d'une beauté simple et sidérante:

nous sommes deux comme des ailes

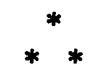

La poésie de Daniel Guénette a de quoi déconcerter. Mais elle s'affirme de plus en plus dans ses qualités de simplicité et d'humour, même si cette simplicité s'entoure volontiers encore d'une gangue de préciosité. Préciosité qui n'est, du reste, nullement une afféterie. Elle est le fait d'une inspiration à la fois riche et savante, qui peut rappeler celle des Décadents. La Part de l'ode ${ }^{5}$, déjà par son titre, laisse entrevoir que la matière première du discours poétique est moins le monde que la littérature; et que sa modernité, curieusement, ne dédaigne pas de faire appel à des éléments de tradition qui sont passablement recherchés. L'ode est une forme poétique lourde, hypercodée, qu'affectionnaient les classiques et que les romantiques ont mise au rancart. Il y a, chez Guénette, 
par-delà les Décadents, une référence au Baroque. Sa phrase poétique est complexe, recourt à des passés simples fort littéraires, à des inversions que ne motive aucune contrainte de versification, à des subordonnées nombreuses qui relèvent d'une syntaxe raffinée, à des allégories parfois d'inspiration mythologique ou évangélique, à des allusions littéraires qui ne manquent pas de subtilité ([...] elles atteignent des félicités de cours simples).

Pourquoi ce déploiement de ressources? Pour donner, à l'expression, la même richesse d'expression que possède le réel. Car c'est bien du vécu non seulement individuel mais universel, du monde avec ses étages de vérités juxtaposées, ses strates de la connaissance, ses réseaux informateurs, c'est de ce feuilleté immense redoublé par les constructions de Babil ou de Babel, que le poète veut rendre compte. Et cette collecte de sens qui passe par les rhétorique et géométrie n'exclut pas les savoirs plus pratiques ou magiques, la roue inventée les ailes des moulins à vent [...] le jeu d'échecs les cerfs-volants d'orient / carré magique même le bouton à quatre trous. Les mythes euxmêmes, chrétiens et païens, la tradition sont requis pour l'édification du savoir car ce qui s'en perd / pour la mémoire est oubli de soi.

Il n'en reste pas moins que la jonction entre la vérité individuelle et les vastes perspectives humaines n'est pas facile à réaliser et le poème semble parfois sombrer dans l'arbitraire. Guénette en est conscient:

\section{Depuis la colline surplombant villes et champs notre point de vue sur ce qui nous constitue sans doute en vaut plus d' un mais n'est-il pas vrai que notre regard se perd somme toute limité par cette vastitude?}

L'aspect outrancièrement singulier de certains développements est l'effet d'un point de vue sur soi trop absolu, qui a perdu le sens des vérités communicables. Heureusement, Guénette a découvert que les choses peuvent exister simplement, et il ajuste de mieux en mieux son regard aux réalités de ce monde.

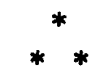

Tirer au clair, de Paul Chanel Malenfant ${ }^{6}$, ne porte pas très bien son titre. $\mathrm{Si}$, en fait, le poète se tourne vers les lieux obscurs de son passé familial pour y prélever des figures et des scènes lourdes d'affects, se livrant à une sorte d'autoanalyse par l'écriture, il semble rejeter vite ses captures dans l'obscurité d'où il les a tirées. En effet, le référent exhibé est soigneusement rendu méconnaissable par tout un travail de brouillage. Père, mère, frère, sœurs deviennent les figurants d'un drame indécidable, dans lequel ils occupent des positions impossibles à démêler. Le bénéfice de l'opération est ainsi perdu, autant pour la poésie que pour l'exorcisme. Peut-être les mobiles de l'élucidation manquent-ils de cette générosité sans laquelle aucun retour sur soi n'est viable. Il s'agit juste de l'angle de tir juste, dit l'un des titres de section, ce qui confere à l'expression tirer au clair une connotation passablement brutale. 
Sur la base d'un énorme malaise individuel et collectif (surtout familial), le poète construit un édifice de langage qui ne manque certes pas de vigueur, ni de beauté. On aimerait toutefois que l'émotion affleure plus directement, que le calcul des effets soit moins présent. Par exemple, la première section se termine par douze poèmes qui comptent chaque fois un vers de moins (de douze à un), et le nombre de vers trouve un écho, direct ou indirect, dans le texte; le poème de neuf vers commence par: Neuf mois de malheur au ventre de goût [...], le poème de quatre vers par: Quatre as entre les lignes les linges [...]; le poème de dix vers évoque les dits commandements (sic), celui de huit vers la faveur de la nuit, celui de sept vers un cerf-volant (c'est moi qui souligne), etc. L'inspiration qui faisait le prix des recueils précédents, depuis le remarquable En tout état de corps $^{7}$, est exploitée de façon certes habile mais un peu trop systématique et voulue. Il y manque de la fraîcheur et peut-être de la nuance:

$J$ ' invoque, pour le désastre, les formes

les plus parfaites, les plus extrêmes.

Admirable, tout de même, de travail et de facture.

Dans Monème, Pierre Desruisseaux ${ }^{8}$ pratique une forme concise, non pour suggérer quelque universelle déficience de l'être mais, au contraire, la densité des choses et des gestes. Une densité fondée en nature, chez cet homme qui rêve de forêts, d'enfance, de pays et de la voix bleue du réel. On retrouve le discours d'un certain humanisme, mais sobre et comme assourdi, rendu tempérant par une sorte de prudence ou de pudeur. Il semble que la simplicité du verbe hésite à se prolonger en réelle beauté littéraire, laquelle suppose un sens accompli. L'image refuse l'éclat, au profit d'un mystère discret et qui décourage l'interrogation:

\section{Perte avant tout \\ $q u$ ' une main sur la pierre \\ cherche l'usage \\ nulle rue neige \\ depuis ne cueille ta présence}

Ce poème réunit bien les vertus de sobriété, de résonance mesurée, de relatif enchantement verbal créé par les mots allitérants (perte / pierre / présence; usage / nulle rue neige). Le poème semble dire une déception (perte; présence non cueillie), et la dit en termes qui affirment la présence, la substance (main sur la pierre). Mais tout cela se referme vite sur le silence. On a l'impression d'un hermétisme par défaut, presque d'une recette. C'est certainement le cas quand le poème verse dans la trivialité - comme celui qui se termine ainsi:

Deux yeux troués cherchent la pharmacie. 
Accident... rare, heureusement. Aussi rare que les réussites évidentes, incontestables ([...] et c' est la nuit allaitée d'os). Le plus souvent, le poème se maintient dans une zone indécidable - farouche, tout près d'être communicable.

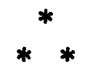

Si l'on en croit la note biographique, il a fallu près de dix ans à Suzy Turcotte pour écrire les poèmes, en vers et en prose, qui composent son premier recueil, De l'envers du corps 9 . Il ne s'agit pourtant pas d'un recueil où chaque vers, chaque mot serait capital, objet d'une décision longuement mûrie, comme ceux de Guy Gervais, de Jean-Pierre Issenhuth, de Robert Melançon ou de Gilles Cyr. Il y a de la spontanéité, de la fraîcheur dans ces effusions, étonnamment chantantes malgré la souffrance ou l'angoisse dont elles témoignent. La simplicité de l'expression frôle parfois la facilité, lorsque l'appel se fait trop direct, bousculant les bienséances littéraires:

Au secours quelqu' un

je ne peux entrevoir

l'envers l'absolue soif

je vais m'écrouler

aveugle

où puiser les forces vives?

Et cette plainte fait bien le fond de tout le recueil, lui donnant sa qualité d'émotion et d'âpreté. Un événement a condamné le poète à ne plus vivre que l'envers des choses: 20 mars 1978: matin pesant où je perdis pied. Depuis, il s'agit de refaire l'unité du corps en morceaux et, par là, accéder au printemps. Le symbolisme, dira-t-on, est peu recherché, mais la vérité du propos s'en accommode. Certes, dans le projet autobiographique, Suzy Turcotte n'atteint pas au lyrisme bouleversant d'une Marie Uguay; néanmoins ses poèmes sont pleins d'un certain bonheur de dire, même s'ils sont au service du petit moi et des amers paysages de sa désolation.

Deux recueils, Brescia, miracle de la justice amère et la Vie est un rêve déjà terminé, me font découvrir une voix grave et juste, qui a plus de présence que nombre d'autres dans le concert des parutions courantes: celle d'Yves Gosselin 10 .

Ce que ces deux livres donnent à lire, c'est une poésie qu'on peut d'abord qualifier de responsable, en ce sens qu'elle ne vise pas à provoquer le vertige mais à découvrir une voie praticable, parmi les ruines qui noircissent l'avenir (Brescia). Gosselin est quelqu'un pour qui le salut existe, de même que la 
justice, l'amour; et la poésie lui apparaît comme l'arme la plus apte à résister aux forces de destruction, aux guerres éternelles (la Vie est un rêve déjà terminé).

Bien entendu, la mauvaise littérature, comme l'enfer, est pavée de bonnes intentions; et la poésie actuelle ne nous a guère habitués aux préoccupations morales. Pourtant, s'il est vrai que la poésie peut tout dire, rien ne l'empêche de prendre en charge la dimension éthique - laquelle est d'autant plus enracinée dans nos vies qu'elle fait l'objet de refoulements plus brutaux.

Je trouve remarquable, en tout cas, l'équilibre que crée Gosselin entre le propos humaniste qui, enraciné dans une problématique personnelle, ne verse jamais dans l'abstraction ou dans la fausse universalité; et l'image, d'une grande robustesse et d'une lisibilité qui n'exclut pas la densité:

Je ne sais toujours pas aimer mes limites perdues

Je ne sais toujours pas si éternellement je vivrai

Aimant sans recours

Séparé de la terre

II me fut donné de vivre dans un espace foudroyé

Où l'éclair martyrisait sa proie

Je sais aujourd' hui mourir

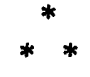

Où est la révolte dans l'Enfant rebelle que publie Michel Muir ${ }^{11}$ ? En fait, les poèmes respirent plutôt l'exaltation, dont l'auteur de Poètes ou imposteurs? et de Poésie: la Noblesse du Réel ${ }^{12}$ a fait sa marque de commerce. Mais curieusement, la frénésie est ici tempérée par un certain sens de la mesure et une sérénité. Point de débauche de points d'exclamation, point de ces intempérances de langage, si fréquentes chez l'essayiste. Le lecteur peut se laisser aller à goûter les fraîches évocations de mes enfants doux et beaux comme des dimanches, ou d'une voix de poivre et de piment et d'azur et de gouffre, même si la vision du monde du poète est carrément de droite. En exergue, une citation de Louis Pauwels condamne l'homme historique au nom de l'homme éternel. Michel Muir, à sa suite, voit dans son siècle un égout, un cloaque de désespoirs où l'on hume avec délectation son remugle de sueurs viciées. Mais dès qu'il se détourne de ses contemporains, le poète devient disponible pour la joie et il arrive que son expression convainque. Pas toujours; certains transferts de classe, comme la formation d'un verbe à partir d'un substantif, procédé cher à Michel Garneau qui y excelle, ne sont guère heureux: les loups [...] vigilent au bord des paupières, le souvenir montagne [...] le jardin de l' intime, etc. L'expression manque parfois de vigueur dans l'invention. Mais il arrive que l'état de grâce s'installe: Moi, témoin cloué, j' explose, poing de fleurs!

L'exaltation, alors, devient enchantement. 
Parmi les ouvrages récents qui gravitent autour de la poésie, citons le remarquable essai de Louise Dupré, Statégies du vertige ${ }^{13}$, qui a valu à son auteur un doctorat de l'Université de Montréal. C'est une étude pénétrante et tout de même accessible sur la poésie de trois Québécoises, Nicole Brossard, Madeleine Gagnon et France Théoret. L'auteur y fait une utilisation exemplaire des concepts de la modernité à des fins de critique littéraire.

Dans un ouvrage qui couvre toute la poésie québécoise des origines à nos jours, Jean Royer fait une présentation sympathique et aussi juste que possible, en si peu de pages, des auteurs regroupés par courants et affinités. Introduction à la poésie québécoise ${ }^{14}$ présente le singulier mérite d'accorder plus de place aux poètes d'aujourd'hui qu'à ceux d'autrefois.

La Nouvelle Barre du jour publie une revue, Première Ligne, dont le numéro d'avril 1989 est entièrement consacré à nos Femmes de lettres ${ }^{15}$. On y trouve plusieurs des voix les plus représentatives de la littérature féminine actuelle.

La jeune maison d'édition le Nordir, fondée au Collège universitaire de Hearst en 1988, publie une revue, Atmosphères, qui consacre sa deuxième livraison à la Littérature franco-ontarienne: poésies, nouvelles, réflexions ${ }^{16}$.

Pour finir, je signale sans commentaire la parution de mon recueil de poèmes intitulé les Matins nus, le vent ${ }^{17}$. C'est un recueil qui... que...

1 Guy Ducharme, Chemins vacants, Montréal, l'Hexagone, 1988, 80 p.

2 Gérald Godin, Poèmes de route, Montréal, l'Hexagone, 1988, 58 p.

3 Renaud Longchamps, Légendes suivi de Sommations sur l'histoire, Montréal, VLB éditeur, 1988, 128 p. Prix Émile-Nelligan 1988.

4 Hugues Corriveau, Apprendre à écrire, Montréal, les Herbes rouges, 1988, 94 p. Signalons que, aux mêmes éditions, l'auteur a fait paraître un roman tout à fait remarquable, les Chevaux de Malaparte (1988, 220 p.).

5 Daniel Guénette, la Part de l'ode, Saint-Lambert, le Noroît/la Bartavelle, 1988, $78 \mathrm{p}$.

6 Paul Chanel Malenfant, Tirer au clair, Saint-Lambert, le Noroît, 1988, 116 p.

7 Paul Chanel Malenfant, En tout état de corps, Trois-Rivières, Ecrits des Forges, 1985. Voir mon compte rendu dans Volx \& images, vol. XI, no 3, printemps 1986, p. 558-559.

8 Pierre Desruisseaux, Monème, Montréal, l'Hexagone, 1989, 122 p. (Poésie).

9 Suzy Turcotte, De l'envers du corps, Montréal, l'Hexagone, 1989, 102 p. (Poésie).

10 Yves Gosselin, Brescia, miracle de la justice amère, Montréal, Triptyque, 1987 82 p., et la Vie est un rêve déjà terminé, Hearst, le Nordir, 1988, 60 p. (Poésie). Yves Gosselin a aussi publié chez Triptyque Connaissance de la mort (1988) qui ne m'est pas parvenu.

11 Michel Muir, l'Enfant rebelle, Hearst, le Nordir, 76 p. (Poésie).

12 Michel Muir, Poètes ou imposteurs?, Verdun, Louise Courteau éditrice, 1985, 180 p., et Poésie: la Noblesse du Réel, Verdun, Louise Courteau éditrice, 1988, 200 p.

13 Louise Dupré, Stratégies du vertige, Montréal, les Éditions du Remue-ménage, $1989,266 \mathrm{p}$. 
14 Jean Royer, Introduction à la poésie québécoise, Montréal, Bibliothèque québécoise, $1989,296 \mathrm{p}$.

15 Femmes de lettres dans Première Ligne, Montréal, la Nouvelle Barre du jour, avril 1989, $116 \mathrm{p}$.

16 Littérature franco-ontarienne: poésies, nouvelles, réflexions dans Atmosphères, no 2, Hearst, le Nordir, 1989,70 p.

17 André Brochu, les Matins nus, le vent, Montréal, Trois, 1989, 80 p. 